\title{
Role of Anti-Cyclic Citrullinated Peptide Antibodies and Rheumatoid Factor in the Diagnosis and Pharmacological Treatment of Rheumatoid Arthritis
}

\author{
Rubén Clavero ${ }^{1}$, Ana Isabel Navarro ${ }^{2}$, Mar Ruiz ${ }^{2}$ and José Miguel Urra ${ }^{1,2 *}$ \\ ${ }^{1}$ Facultad de Medicina de Ciudad Real, Universidad de Castilla La Mancha (UCLM), España, Spain \\ ${ }^{2}$ Laboratorio de Inmunología, Hospital General Universitario de Ciudad Real, España, Spain
}

\begin{abstract}
Two characteristic autoantibodies are tested for the diagnosis of Rheumatoid Arthritis (RA); the rheumatoid factor (RF) and anti-cyclic citrullinated peptide antibodies (CCP). The presence of these antibodies determines that RA is classified as seropositive or seronegative, with important implications both in the clinical development and in the prognosis of the disease. It is interesting to know the role that both, CCPs and RF, play in the diagnosis of RA, as well as the influence that the design of these tests have on the results obtained.

RF and CCP were tested in 213 patients with an initial clinical suspicion of RA. Based on the analytical results and in the clinical parameters a definitive diagnosis was obtained. A total of 63 of the 213 patients (29.58\%) had a definitive diagnosis of RA, of which $77.8 \%$ were classified as seropositive by the presence of at least one of the two autoantibodies analyzed.

The sensitivity and specificity were higher for the CCP than the RF. Initially, the RF showed a low diagnostic value since was negative in more than two thirds of the patients with a definitive RA diagnosis. A precise adjustment of the cut-off value in RF detection from a study in healthy population was necessary to be able to use this test as a useful diagnostic tool.

The presence of antibodies in patients with RA affect to the treatment of choice with greater use of Methotrexate compared to other immunosuppressants in seropositive group of RA patients and a higher proportion of patients with other immunosuppressants in the group of seronegative patients.

In conclusion, the CCP presented better diagnostic test validity parameters for RA diagnosis than the RF. An adequate cut-off point in the detection of antibodies is essential for validation as useful diagnostic tools. The presence of antibodies in RA influences the treatment of choice, possibly due to the greater severity of the pathology.
\end{abstract}

\section{Keywords}

Rheumatoid Arthritis, Anti-Cyclic Citrullinated Peptide Antibodies, Rheumatoid Factor

\section{Introduction}

Rheumatoid Arthritis (RA) is a chronic systemic disease characterized by irreversible inflammation and destruction of the articular cartilage and underlying bone. The disease follows a progressive course, which if not treated properly can lead to a significant disability [1]. Its main characteristic is the presence of persistent synovial inflammation along with joint damage [2]. RA mainly affects small and medium sized joints. The metacarpophalangeal and proximal interphalangeal joints are the most commonly affected [3]. In very advanced stages of the disease it is when deformities can be observed as a result of joint damage and destruction. Extra-articular

*Corresponding author: José Miguel Urra Ardanaz, Laboratorio de Inmunología, Facultad de Medicina de Ciudad Real, Hospital General Univrsitario de Ciudad Real, 13005, Ciudad Real, España, Spain

Accepted: July 03, 2021

Published online: July 05, 2021

Citation: Clavero R, Navarro AN, Ruiz M, et al. (2021) Role of Anti-Cyclic Citrullinated Peptide Antibodies and Rheumatoid Factor in the Diagnosis and Pharmacological Treatment of Rheumatoid Arthritis. J Phys Med 3(1):66-70 
Citation: Clavero R, Navarro AN, Ruiz M, et al. (2021) Role of Anti-Cyclic Citrullinated Peptide Antibodies and Rheumatoid Factor in the Diagnosis and Pharmacological Treatment of Rheumatoid Arthritis. J Phys Med 3(1):66-70

manifestations usually occur in patients with long-standing RA and severe forms.

Currently, the criteria of the EULAR (European League against Rheumatic Diseases)/ACR (American College of Rheumatology) proposed in 2010 are those used to establish the diagnosis of RA [3]. The current criteria differ from those previously proposed in establishing an earlier diagnosis of the disease and early start of treatment, thus reducing joint destruction and damage. At present there is an arsenal of treatments that allow an adequate control of the disease $[4,5]$.

RA is a disease of autoimmune etiology, in which the two most relevant antibodies for the diagnosis and management of the pathology are the rheumatoid factor (RF) and anticyclic citrullinated peptide antibodies (CCP). In the diagnostic guidelines of the EULAR/ACR 2010 the presence of these autoantibodies was established as diagnostic criterion for RA.

RF was the first antibody described for RA being an autoantibody that bound the constant fraction of IgG [6]. The presence of RF despite its low specificity was already included in the diagnostic criteria of the year 1987 [7]. The CCPs are directed against citrullinated proteins, in which conversion of the aminoacid arginine into citrulline has been produced through an enzymatic citrullination process. This posttranslational modification can be recognized as foreign by the immune system. The CCPs have a high specificity in RA $[8,9]$. These antibodies are very useful in the early stages of the disease in which even though diagnostic criteria for RA are not yet met CCPs are already present. Among these patients, the vast majority (90\%) will develop RA in the next three years, so CCPs are very good predictors of disease [4]. Together with early detection, these antibodies have also been related to the course of the disease and their concentration has been shown to determine the degree of bone destruction [10].

Within the RA, a subdivision has been established based on the presence (seropositive) or absence (seronegative) of the autoantibodies (RF and/or CCP). These two entities are considered different in terms of disease progression, joint destruction and even treatment [11]. Seropositive subjects, and especially those with CCP, have a more marked progression of bone erosion and destruction than seronegative [10]. It is known that seropositive patients have a better response to methotrexate (MTX), while seronegative patients do not have such a good response to the same treatment [12]. The main objective of the present work is to evaluate the role of CCP and RF antibodies in the diagnosis of RA and its relationship with the established pharmacological treatments.

\section{Material and Methods}

\section{Patients}

All patients with a clinical suspicion of RA in the period between February-April 2019 who were tested for RA $t$ antibodies are included in the study. Patients who for any reason could not collect all the variables designed in the study were excluded. Finally, 213 patients were analyzed. The variables were collected from its clinical histories and biochemical/immunological findings. The following variables were collected: sex, age, results of RF and CCP, final diagnosis, and in patients with a diagnosis of RA, the serogroup of the disease (seropositive or seronegative RA), as well as the treatment established, classified as follows: Methotrexate (MTX), anti TNF $\alpha$ (adalimumab, certolizumab, golimumab, etanercept), and the rest of the disease modifying treatments included in the group called "Others" (hydroxychloroquine, leflunomide ...).

\section{RF determination}

The method used measures the concentration of serum RF by latex-enhanced immunoturbidimetry (ADVIA XPT 2400 Siemens Healthcare, GER). Positive for RF was considered if the plasma concentration of the patients was $>60 \mathrm{IU} / \mathrm{mL}$. This reference value is set and used by the laboratory according to the recommendations of the reagent supply company.

\section{CCP assessment}

The presence of CCP was determined by a quantitative enzyme immunoassay on wells coated with peptides containing arginine residues modified by citrullination (Euroimmun, Lübeck GER). As a cut-off point, the recommended by the supplier was used, considering the presence of CCP when the value obtained is $>5 \mathrm{U} / \mathrm{mL}$.

\section{Statistical analysis}

For the assessment of the diagnostic efficacy parameters of RF and CCP, false negatives (FN), false positives (FP), true negatives (TN) and true positives (TP) were identified. From these data, the following parameters were calculated as diagnostic efficacy parameters: Sensitivity (S): $S=T P /(T P+$ FN); Specificity $(E): E=T N /(T N+F P)$; Positive predictive value $(P P V): P P V=T P /(T P+F P) ;$ Negative predictive value (NPV): $V P N=T N /(F N+T N)$.

The associations between qualitative parameters were studied using the Pearson's $\chi^{2}$ Test, using Fisher's Exact Test in the cases where it was necessary (observed and/or expected $<5$ ). For the comparison of quantitative parameters, the Student's T test was used for independent samples. Significant results were considered to be those that obtained a $p$-value of less than 0.05 . SPSS Statistics version 25 has been used for statistical data processing.

\section{Results}

\section{Patients}

During the study period, 213 blood samples of patients were analyzed for CCP and RF. None of them had previous studies. For both antibodies 203 patients were tested, while the remaining 10 were only checked for CCPs. Of the total of the sample studied, 63 patients (29.58\%) corresponded to subjects with a definitive diagnosis of RA according to the ACR criteria, while the rest 150 patients $(70.42 \%)$ did not meet the criteria diagnosis of the disease. The demographic data of the patients are shown in Table 1. It highlights a higher frequency of female sex in RA diagnosed patients (68.2\%), and also with an earlier onset of the disease, approximately seven years earlier than men. 
Citation: Clavero R, Navarro AN, Ruiz M, et al. (2021) Role of Anti-Cyclic Citrullinated Peptide Antibodies and Rheumatoid Factor in the Diagnosis and Pharmacological Treatment of Rheumatoid Arthritis. J Phys Med 3(1):66-70

\section{Antibody studies}

The results obtained in the studies of both antibodies based on the diagnosis of RA are presented in Table 2. The results of the diagnostic validity parameters obtained from the definitive diagnoses shown in Table 3. The results obtained for the CCPs are consistent with those described in other series, however the results obtained for the RF show a very low sensitivity, below $35 \%$. From the present data it is deduced that the RF test, by itself, discards a large number of potential patients with RA (false negatives).

The seropositive RA is defined as having a positive result for one or both of the two autoantibodies studied. Of the 63 patients with the diagnosis of RA, it was determined that 49 of them $(77.8 \%)$ correspond to seropositive RA, while $14(22.2 \%)$ belong to seronegative RA (Table 1 ). The female frequency was higher in both groups. A certain predisposition was observed with younger patients in the seropositive, but without statistical significance $(p=0.61)$.

\section{$\mathrm{RF}$ reference values}

Due to the low sensitivity results obtained for the RF, it was deduced that the recommended reference value was excessively high. The appropriate value for our population was determined. For this purpose, the determination was made in 150 healthy individuals in which any type of clinic and family history related to connective disease was previously ruled out. The mean RF obtained was $9.4 \mathrm{IU} / \mathrm{mL} \pm$ 1.6 (standard deviation). From this data, two reference values

Table 1: Demographic data of the patients included in the study. Data are shown based on the diagnosis of RA and if the disease is classified as seropositive or seronegative based on the presence of antibodies (CCP and/or RF). Sex is presented in percentages and age in years \pm standard deviation.

\section{PATIENTS UNDER STUDY}

\section{$N=213$}

\begin{tabular}{l|l} 
No RA & RA
\end{tabular}

$150(70.42 \%) N \quad 63(29.58 \%)$

\begin{tabular}{|l|l|l|l|}
\hline & Age & $56.27 \pm 14.34$ & \\
\hline & Sex & Male & Female \\
\hline & & $20(31.75 \%)$ & $43(68.25 \%)$ \\
\hline & Age & $61.65 \pm 10.7$ & $53.77 \pm 15.21$ \\
\hline & AR & Seropositive & Seronegative \\
\hline & N & $49(77.8 \%)$ & $14(22.2 \%)$ \\
\hline & Sex & $71.4 \%$ Female & $67.3 \%$ Female \\
\hline & Age & $55.6 \pm 13$ & $58.43 \pm 18.7$ \\
\hline
\end{tabular}

Table 2: Results of the RF and CCP autoantibodies in relation to the diagnosis of RA. The results are expressed in absolute number and percentages.

\begin{tabular}{|c|c|c|c|}
\hline \multicolumn{2}{|c|}{ RA } & no RA & Total \\
\hline CCP + & $49(78 \%)$ & $9(6 \%)$ & 58 \\
\hline CCP - & $14(22 \%)$ & $141(94 \%)$ & 155 \\
\hline & 63 & 150 & 213 \\
\hline & RA & no RA & Total \\
\hline RF + & $22(35 \%)$ & $8(6 \%)$ & 30 \\
\hline RF - & $41(65 \%)$ & $132(94 \%)$ & 173 \\
\hline & 63 & 140 & 203 \\
\hline
\end{tabular}

Table 3: Parameters of diagnostic efficacy of RF and CCP antibodies. The results are expressed in percentages.

\begin{tabular}{|l|c|c|c|c|}
\hline & SENSIBILITY & SPECIFIDCITY & PPV & NPV \\
\hline CCP & 77.8 & 94.0 & 84.0 & 91.0 \\
\hline RF & 34.9 & 94.3 & 73.3 & 76.3 \\
\hline
\end{tabular}

Table 4: Analysis of the parameters of diagnostic efficiency of the RF according to the reference values. The first $60 \mathrm{U} / \mathrm{mL}$ is recommended by the reagent supplier, the second $18 \mathrm{U} / \mathrm{mL}$ is obtained from the 97.5 percentile from the average obtained in a population of 150 healthy individuals, and the third $12.5 \mathrm{U} / \mathrm{mL}$ corresponds to the mean \pm two standard deviations of the results obtained in the same population. The results are expressed in percentages.

\begin{tabular}{|l|c|c|c|c|}
\hline Cut Off & $\begin{array}{c}\text { Sensibility } \\
\text { (\%) }\end{array}$ & $\begin{array}{c}\text { Specificity } \\
\text { (\%) }\end{array}$ & PPV (\%) & NPV (\%) \\
\hline $\mathbf{6 0 ~ U I / m L}$ & 34.9 & 94.3 & 84.0 & 91.0 \\
\hline $\mathbf{1 8} \mathbf{U I} / \mathbf{m L}$ & 55.5 & 87.9 & 67.3 & 81.4 \\
\hline $\mathbf{1 2 . 5} \mathbf{~ U I / m L}$ & 65.0 & 83.6 & 64.0 & 84.2 \\
\hline
\end{tabular}

Table 5: Frequencies of the different diagnoses obtained from patients with false positive RF and CCP antibodies. The results are expressed in percentages.

\begin{tabular}{|l|c|c|}
\hline$\%$ & CCP & RF \\
\hline RA & 84 & 73 \\
\hline PROBABLE RA & 8 & 3 \\
\hline POLYARTHRITIS & 2 & 0 \\
\hline RIZARTROSIS & 2 & 0 \\
\hline SJORGREN & 2 & 3 \\
\hline LUMBALGIA & 2 & 3 \\
\hline HAND ARTRALGY & 0 & 7 \\
\hline CUTANEOUS LUPUS & 0 & 3 \\
\hline KNEE PAIN GONALGIA & 0 & 4 \\
\hline ARTROSIS & 0 & 4 \\
\hline
\end{tabular}

were obtained, using the 97.5 percentile $(18 \mathrm{IU} / \mathrm{mL})$ and considering the mean \pm 2 standard deviations $(12.5 \mathrm{IU} / \mathrm{mL})$. With these two new reference values, results were obtained for RF with more adequate sensitivities for the diagnosis of RA as show in Table 4. The changes in the reference value did not affect the determination of seropositivity in RA since all patients with positive RF with the new reference values already had positive CCPs.

\section{Antibodies in entities other than RA}

Patients with CCP mostly correspond to the diagnosis of RA (84\%). However, there is a percentage of patients that does not correspond to this entity. Many of these diagnoses are not fully established and have been classified as "probable RA," or polyarthritis, (Table 5). The probable RA correspond to entities that without completing the EULAR 2010 diagnostic criteria for RA, are considered probable both for their clinical manifestations and for their debut. A large part of them will end up developing the disease in the future.

In patients with RF+ the percentage with a diagnosis of RA is lower than in CCP (73\%), however the diversity of pathologies associated with this antibody is higher. We can find clearly defined diseases, such as Sjögren's syndrome, cutaneous lupus or osteoarthritis, but also other pathologies with a poor 
Citation: Clavero R, Navarro AN, Ruiz M, et al. (2021) Role of Anti-Cyclic Citrullinated Peptide Antibodies and Rheumatoid Factor in the Diagnosis and Pharmacological Treatment of Rheumatoid Arthritis. J Phys Med 3(1):66-70

concise diagnosis, as is the case with $\mathrm{CCP}+$ patients. Among these we can highlight arthralgia of hands, and another group of entities classified as "probable RA" (Table 5).

\section{Treatments based on the presence of antibodies}

Regarding the treatment used for RA, 25 patients (39.68\%) are treated with MTX alone, 11 with anti TNF alone (17.46\%), 11 with a combination of MTX and anti TNF (17.46\%) and $16(25.4 \%)$ with other drugs such as leflunomide, hydroxychloroquine. Corticosteroids have been excluded along with NSAIDs as they are used to control acute episodes of the disease in combination with any of the interior drugs.

If we distinguish between seropositive and seronegative RA subjects, almost half (45\%) of seropositive patients use MTX monotherapy compared to $21 \%$ of subjects with seronegative RA. In contrast, in seronegative patients, the "other drugs" group is used more in decrease of MTX as shown in Figure 1. No statistically significant differences were observed, although there was a tendency towards a greater use of MTX compared to other drugs in the case of seropositive RA $(p=0.1)$.

\section{Discussion}

The early detection of RA is essential because with an early stage action of the disease, the progressive course of the disease can be avoided and the disabling aftermath that it generates as a result of irreversible bone ersions can be prevented [1]. The detection of antibodies associated with RA can be a useful tool for an early diagnosis and the initiation of an adequate treatment. Antibodies have been shown to precede the disease and can be detected even 10 years before clinical manifestations [8]. The presence of the antibodies together with the inconclusive clinical manifestations suggests the so-called "undifferentiated arthritis". These patients would require follow-up to be able to diagnose the disease as soon as possible or even to start a treatment to reduce the progression of RA if necessary. From all the aforementioned, it is concluded that it is very important to know the diagnostic value that RF and CCP present in RA. In seronegative RA there is currently no possibility of detecting the disease early with the tests used. Therefore, it would be of great interest to deepen more about other antibodies that have been detected in RA such as antibodies against acetylated and carbamylated proteins $[13,14]$ since these could be present in what we currently call seronegative RA.

The results presented for CCP are consistent with that described in other series [15], but instead those obtained by RF showed a very low sensitivity to be used as an early diagnostic tool. When establishing a high cut-off point, according to the recommendation of the supplying company, the specificity was much higher than previously described [16], while its sensitivity was much lower, excluding two thirds of potential RA patients with adequate results. Given these results, we chose to adjust the reference value of the technique based on our general population. Using a suitable reference value, a sensitivity similar to that of the CCPs was achieved although with a lower specificity as described in other series, and in this way the RF becomes a useful tool for a diagnosis of RA.

Patients with false positive results were higher in the RF $(27 \%)$ than CCP (16\%). In addition to the false positive assumptions, many of them in the case of CCPs correspond to possible RA. The presence of these antibodies probably suggests the so-called undifferentiated arthritis, so it is expected that most of these patients will develop RA within the next 3 years [4]. On the contrary we can find RF in other clearly defined pathologies such as Sjögren's syndrome, osteoarthritis, mechanical gonalgia, cutaneous lupus in a greater proportion than the CCP. Finally, if we consider the treatment used for RA, we must emphasize that the fundamental drug is MTX. However, its use differs in relation to the RA serotype. In almost half of seropositive

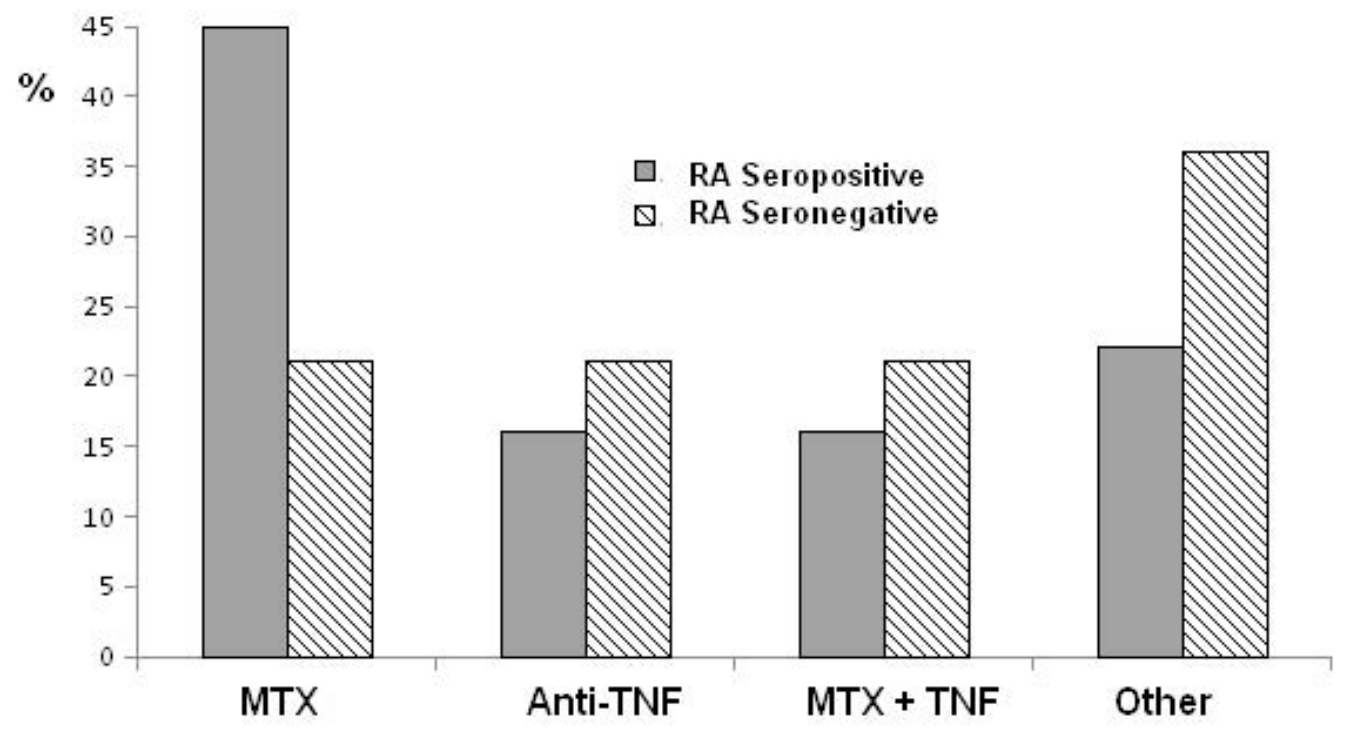

Figure 1: Percentage of treatments implanted in RA patients according to the classification of the disease in seropositive or seronegative according to the presence of CCP and/or RF antibodies. Methotrexate (MTX), Biological (anti-TNF $\alpha$ ), Other (leflunomide, hydroxychloroquine). 
Citation: Clavero R, Navarro AN, Ruiz M, et al. (2021) Role of Anti-Cyclic Citrullinated Peptide Antibodies and Rheumatoid Factor in the Diagnosis and Pharmacological Treatment of Rheumatoid Arthritis. J Phys Med 3(1):66-70

individuals, the disease can be controlled with MTX alone. This percentage is higher if we consider that in case MTX is not enough, another drug will be added to it to control the disease. In the case of seronegative RA, the percentage of MTX use decreases to $21 \%$. This may be due to the fact that in these individuals the disease can be controlled with the use of other immunomodulators, since these patients follow a totally different course and generally the disease does not have such an aggressive character as the seropositive RA [12]. Therefore, it can be established that in seropositive RA a better response is achieved with MTX, either as monotherapy or in combination with other drugs. On the contrary, in seronegative RA the treatment is more diverse, since there is no clear predilection for any of the drugs.

\section{Declaration of Interest}

The authors certify that they have NO affiliations with or involvement in any organization or entity with any financial interest in the subject matter or materials discussed in this manuscript.

\section{References}

1. Scott DL, Wolfe F, Huizinga TW (2010) Rheumatoid arthritis. Lancet 376: 1094-1108.

2. Van Oosterhout M, Bajema I, Levarht EWN, et al. (2008) Differences in synovial tissue infiltrates between anti-cyclic citrullinated peptide-positive rheumatoid arthritis and anticyclic citrullinated peptide-negative rheumatoid arthritis. Arthritis Rheum 58: 53-60.

3. Aletaha D, Neogi T, Silman AJ, et al. (2010) F2010 Rheumatoid arthritis classification criteria: An American College of Rheumatology/European League Against Rheumatism collaborative initiative. Arthritis Rheum 62: 2569-2581.

4. Van der Helm-vanMil AHM, le Cessie $\mathrm{S}$, van Dongen $\mathrm{H}$, et al. (2007) A prediction rule for disease outcome in patients with Recent-onset undifferentiated arthritis: How to guide individual treatment decisions. Arthritis Rheum 56: 433-440.

5. Wunderlich C, Oliviera I, Figueiredo CP, et al. (2017) Effects of DMARDs on citrullinated peptide autoantibody levels in RA patients-A longitudinal analysis. Semin Arthritis Rheum. Elsevier 46: 709-714.
6. Anquetil F, Clavel C, Offer G, et al. (2015) IgM and IgA rheumatoid factors purified from rheumatoid arthritis sera boost the $\mathrm{Fc}$ receptor- and complement-dependent effector functions of the disease-specific anti-citrullinated protein autoantibodies. J Immunol 194: 3664-3674.

7. Arnett FC, Edworthy SM, Bloch DA, et al. (1988) The American Rheumatism Association 1987 revised criteria for the classification of rheumatoid arthritis. Arthritis Rheum 31: 315324.

8. Nielen MMJ, van Schaardenburg D, Reesink HW, et al. (2004) Specific autoantibodies precede the symptoms of rheumatoid arthritis: A study of serial measurements in blood donors. Arthritis Rheum 50: 380-386.

9. Zendman AJW, van Venrooij WJ, Pruijn GJM (2006) Use and significance of anti-CCP autoantibodies in rheumatoid arthritis. Rheumatology 45: 20-25.

10. van der Helm-van Mil AH, Verpoort KN, Breedveld FC, et al. (2005) Antibodies to citrullinated proteins and differences in clinical progression of rheumatoid arthritis. Arthritis Res Ther 7: 949-958.

11. Derksen VFAM, Ajeganova S, Trouw LA, et al. (2017) Rheumatoid arthritis phenotype at presentation differs depending on the number of autoantibodies present. Ann Rheum Dis 76: 716-720.

12. van Dongen $H$, van Aken J, Lard LR, et al. (2007) Efficacy of methotrexate treatment in patients with probable rheumatoid arthritis: A double-blind, randomized, placebo-controlled trial. Arthritis Rheum 56: 1424-1432.

13. Juarez $M$, Bang $H$, Hammar F, et al. (2016) Identification of novel antiacetylated vimentin antibodies in patients with early inflammatory arthritis. Ann Rheum Dis 75: 1099-1107.

14. Wang Z, Nicholls SJ, Rodriguez ER, et al. (2007) Protein carbamylation links inflammation, smoking, uremia and atherogenesis. Nat Med 13: 1176-1184.

15. Enriconi dos Anjos LM, Pereira IA, d 'Orsi E, et al. (2009) A comparative study of IgG second- and third-generation anticyclic citrullinated peptide (CCP) ELISAs and their combination with IgA third-generation CCP ELISA for the diagnosis of rheumatoid arthritis. Clin Rheumatol 28: 153-158.

16. Nielsen SF, Bojesen SE, Schnohr P NB, et al. (2012) Elevated rheumatoid factor and long term risk of rheumatoid arthritis: $A$ prospective cohort study. BMJ 345: e5244.

DOI: $10.36959 / 942 / 342$

Copyright: (C) 2021 Clavero R. This is an open-access article distributed under the terms of the Creative Commons Attribution License, which permits unrestricted use, distribution, and reproduction in any medium, provided the original author and source are credited. 